\title{
El peligro del adoctrinamiento en la enseñanza de la ética y la deontología de la comunicación
}

\author{
Jesús DíAz DEL CAMPo LozANo \\ Universidad Internacional de La Rioja \\ jesus.diaz@unir.net
}

\begin{abstract}
Resumen
Uno de los mayores riesgos que afronta el profesor encargado de enseñar Ética y Deontología a los alumnos de Comunicación es el peligro de incurrir en el adoctrinamiento que puede ser tanto metodológico como de contenido. Este artículo se plantea cuáles son las fórmulas docentes más adecuadas evitar ese riesgo. Para ello, se apoya en los resultados de una investigación llevada a cabo en 14 países miembros de la Unión Europea.
\end{abstract}

Palabras clave: Ética de la Comunicación - Educación moral - Dilemas morales - Profesorado universitario - Adoctrinamiento

The danger of indoctrination in teaching communication ethics and deontology

\begin{abstract}
One of the main risks professors teaching Communication Ethics and Deontology have to deal with is indoctrination, which can be referred both to methodology and content. This paper aims to look for the better pedagogical techniques in order to avoid indoctrination. Moreover, we show the main findings of a survey carried out in 14 European Union member states.
\end{abstract}

Key Words: Communication ethics - Moral Education - Moral Dilemmas - University teachers Indoctrination

\section{Referencia normalizada:}

Díaz del Campo Lozano, J. (2013) El peligro del adoctrinamiento en la enseñanza de la ética y la deontología de la comunicación. Historia y Comunicación Social. Vol. 18 № Especial Octubre. Págs. 331-341.

\section{Sumario}

1. Introducción y marco teórico. 1.1. El adoctrinamiento en la enseñanza de la ética. 2. Metodología. 3. Resultados. 4. Conclusiones y prospectiva. 5. Referencias bibliográficas

\section{Introducción y marco teórico}

Uno de los grandes expertos en ética de la comunicación, el profesor estadounidense John Merrill (1978: 59), planteaba la siguiente cuestión: “¿Realmente enseñamos ética (es decir, lo que los periodistas deben hacer) o enseñamos contenidos referentes a la ética? ¿Intentamos inculcar determinados modelos o principios éticos en los alumnos - o simplemente presentamos una variedad de soluciones éticas alter- 
nativas, dejando que sean los alumnos los que decidan y saquen sus propias conclusiones?".

A buen seguro que son muchos los profesores de la asignatura que se han hecho en algún momento esa misma pregunta, y es que la ética constituye un saber peculiar en el que resulta más sencillo teorizar que llevar algo a la práctica. Es ahí precisamente donde reside su gran paradoja pues, como explicaba Aristóteles (1988: 160), la ética es un saber eminentemente práctico en el que "investigamos no para saber qué es la virtud, sino para ser buenos, ya que de otro modo ningún beneficio sacaríamos de ella". Todo ello comporta una aplicación a los distintos ámbitos y actividades sociales, a los que proporciona un conjunto de referentes o principios para hacer frente a los dilemas que se plantean a quienes ejercen dichas actividades. De ahí que tanto la ética general como las éticas aplicadas son éticas que ya sea en sus principios o en sus directrices buscan el bien de los clientes o usuarios de los servicios, de la sociedad y de los propios profesionales (Cobo, 2001). En consecuencia, esos elementos peculiares a las que se refería Aristóteles tienen como principal consecuencia en el caso de las éticas aplicadas que el "objetivo fundamental no es que los alumnos conozcan lo que es ser un buen profesional sino que acaben siendo buenos profesionales" (Etxeberría, 2000: 311).

Al mismo tiempo, la Ética, al igual que le sucede a cualquier otra disciplina, se compone de una serie de conceptos y métodos clave, pero además, como consecuencia de que su objeto de estudio es el comportamiento del ser humano y el carácter moral, guarda una estrecha relación con el mundo de los valores y, por ello, no puede ser enseñada en el aula con el mismo grado de neutralidad que, por ejemplo, la Física o la Química. De hecho, en último término la Ética no deja de ser una cuestión personal que corresponde a la conciencia individual, lo que implica que no exista una única respuesta para las diversas cuestiones que se plantean en su ámbito de estudio. En otras palabras, y en el universo propio de la Comunicación, dos periodistas que se encuentren en la tesitura de elaborar una información sobre un mismo hecho noticioso pueden tomar decisiones diferentes, basándose para ello en criterios igualmente diferentes, $\mathrm{y}$, sin embargo, será perfectamente posible que tanto una como otra postura resulten correctas desde un punto de vista ético.

Por eso, a la hora de plantearse la enseñanza de la asignatura, surge el dilema entre el planteamiento más teórico-abstracto y un enfoque más casuístico, una cuestión en la que Linde (2009: 57)) apuesta por este segundo método, "basado en la aplicación de principios, en la búsqueda del equilibrio reflexivo en la discusión de dilemas morales, es un buen instrumento para el aprendizaje de la ética y deontología de la comunicación". Sin duda, este enfoque parece más adecuado para evitar el riesgo del adoctrinamiento, cuyas características se examinan a continuación.

\subsection{El adoctrinamiento en la enseñanza de la ética}

En términos generales, el adoctrinamiento puede consistir en la manifestación de algún tipo de prejuicio del profesor ante sus alumnos, pero de manera más rigurosa 
se puede definir como "el objetivo de un profesor que intenta claramente que los alumnos acepten un sistema de creencias cerrado, que excluye la autocrítica, y que excluye sistemáticamente la posibilidad del rechazo" (The Hastings Center, 1980: 59). Se trata de un problema que se puede presentar a cualquier profesor, pero que conlleva una especial importancia en el caso de la ética, debido a que el objeto de estudio es el comportamiento del ser humano -en este caso, el profesional de la comunicación- y a que guarda una relación muy estrecha con el mundo de los valores.

De manera un poco más detallada, el adoctrinamiento se da cuando un profesor intenta persuadir sistemáticamente a sus alumnos de la validez de un sistema de creencias concreto y además (The Hastings Center, 1980: 59):

- Descarta de manera radical la posibilidad de aceptar otros sistemas.

- Busca, deliberadamente, el rechazo de los alumnos tanto a aquellas objeciones serias que se hayan realizado a ese sistema como también a las herramientas analíticas que los capacitarían para comprobar de manera más palpable los errores de ese sistema.

- Excluye la posibilidad de rechazar ese sistema de creencias

- Penaliza a todo aquel que se desvía de ese sistema.

Por tanto, se trata de una postura contraria a la naturaleza misma de la ética, pues el profesor presupone frente a los alumnos que sólo hay un sistema teórico válido y un único conjunto de respuestas verdaderas a los problemas éticos. Al mismo tiempo, coarta cualquier posibilidad de que el futuro profesional pueda desarrollar un conjunto de herramientas y criterios analíticos que le sirvan para tomar las mejores decisiones posibles en el ejercicio de su actividad. Los responsables del Hastings Center (1980: 60-61) añaden otros tipos de adoctrinamiento que resultan a priori menos evidentes, pero que en el fondo constituyen posturas igualmente poco adecuadas de cara a la correcta enseñanza de la asignatura:

- Adoctrinamiento metodológico. Consiste en hacer primar ante los alumnos una metodología y relegar todas las demás. En ocasiones, su aplicación puede llevar a favorecer una teoría ética por encima de otras.

- Sin llegar a los términos tan absolutos, el profesor puede promover la idea de que ciertas cuestiones o teorías éticas son mucho más importantes que otras que se pasan por alto en la clase. En este sentido, es obvio que los alumnos deberían recibir la explicación de las diferentes nociones éticas y, en particular, a aquellas otras versiones que dejarían en evidencia las propias convicciones del profesor.

- Por último, el profesor también podría diseñar un programa de la asignatura que implícitamente conlleve una cierta predilección hacia ciertas teorías o sistemas. Este último supuesto puede llevarse a cabo fácilmente a través de la elección de obras y textos de referencia que los alumnos deberán estudiar o analizar a lo largo del curso. 
Por tanto, los riesgos para el profesor encargado de impartir la asignatura son diversos. Más aún cuando una postura de neutralidad absoluta tampoco parece la posición más aconsejable, ya que puede llevar a la desorientación y/o desinterés del alumno. Por ello el profesor no debe eludir mostrar sus propias creencias, para que sea el propio alumno el que determine si son más o menos correctas, y debe sentirse totalmente libre para hacerlo. Ya que, como recuerda Christians (citado por Elliot, 1984: 41), los alumnos prefieren eso "a alguien que enmascara su opinión. Si te limitas a presentar alternativas una y otra ver, estás intentando ocultar tu propio punto vista, lo que crea un ambiente mucho más incierto". Eso sí, el docente debería en todo momento justificar los argumentos y no simplemente imponerlos. La puesta en práctica de esta idea conlleva una serie de problemas, ya que por un lado, el objetivo último, siguiendo a Aristóteles, es contar con mejores profesionales, pero por otro, en el universo concreto de la Ética, y en las sociedades de hoy en día, cualquier decisión que se tome puede dar pie a la controversia, y estar abierta a diversas interpretaciones.

Se trata de un conflicto que Etxeberría (2000: 314) resuelve recurriendo a la denominada "ética de mínimos, esto es, la ética compartida resultante de la maduración ética de la sociedad, concretamente la ética implicada en los derechos humanos". Actuando de esta manera, la enseñanza de la ética resulta perfectamente posible, y hasta recomendable, ya que existe una gran diferencia entre "ayudar a que los alumnos lleguen a ser agentes morales autónomos e imponerles nuestro propio sistema de valores" (Black, 1992: 235).

En este sentido, no cabe duda de que la actitud del buen periodista debe aproximarse lo máximo posible a la filosofía moral y alejarse de la moralización. Algo para lo que sería positivo que disfrutara de un nivel de autonomía tan alto como sea posible, un objetivo que no obstante resulta sumamente complicado de alcanzar en muchas ocasiones, a tenor de las condiciones en las que ejerce su trabajo diario. De todos modos, es lícito pensar que cuanto mayor sea el avance en ese camino durante el proceso de formación, mejor preparado llegará y más firmemente se podrá enfrentar a todas las presiones citadas. De hecho, "será suficiente que un único periodista individual sepa dónde dibujar la línea y cómo tomar sus decisiones, para que haya merecido la pena enseñar ética" (Merrill, 2002).

En resumen, todas estas cuestiones conducen a formular una cuestión muy concreta: cuál es el método y cuáles son las técnicas pedagógicas más adecuadas para enseñar la Ética y Deontología de la Comunicación. Como condición previa e indispensable parece claro que se tratará de técnicas y métodos que aporten herramientas para realizar esos juicios y resolver los dilemas de la manera más ética posible.

El estudio de casos, a ser posible en pequeños grupos, será el sistema que más se acerque a este ideal. Es una técnica que consiste en el planteamiento de situaciones -hipotéticas o inspiradas directamente en ejemplos que han tenido lugar en la vida real-, que constituyen un dilema entre distintas, a partir de las cuales el alumno intentará tomar una decisión lo más ética posible. Se trata de un método que busca, por tanto, despertar el interés y lograr la implicación y la participación activa de los 
alumnos, para que, en último término, la clase se convierta en un diálogo permanente y no en una simple exposición de conceptos por parte del profesor encargado de impartirla. En este sentido, la actuación diaria de los medios de comunicación proporciona numerosísimos ejemplos para ser estudiados en el aula.

Otros dos métodos pedagógicos cuya utilización puede ser muy pertinente para evitar el adoctrinamiento son el role playing, y la discusión en pequeños grupos. En el primero de ellos, se desarrolla una dinámica que presenta ligeras variaciones respecto al estudio de casos, por cuanto en lugar de que todos los alumnos asuman la función del profesional, el profesor asigna los diversos papeles correspondientes a todas las partes implicadas.

Mientras, con el empleo de la discusión en pequeños grupos se evita que la clase termine siendo un mero intercambio de opiniones personales no siempre bien razonadas, $\mathrm{y}$, del mismo modo, facilita las cosas cuando el dilema que hay que resolver es controvertido, y los alumnos tienden a aludir la responsabilidad (The Hastings Center, 1980: 70). Por tanto, cuando el profesor de la asignatura opta por unas técnicas pedagógicas en detrimento de otras está tomando una decisión que resultará determinante a la hora de evitar el riesgo del adoctrinamiento.

En último término, con un enfoque mucho más amplio, que abarca no sólo la metodología sino la asignatura en sí misma, Elliot (1984: 164-165) plantea un listado de recomendaciones que denominó Responsabilidades del profesor de ética periodística:

- Ser consciente del propio sistema de creencias morales.

- Educar a los alumnos y no adoctrinarlos

- Ayudarlos en la tarea de que comprendan cuáles son las obligaciones básicas derivadas de la función de los medios de comunicación en la sociedad y de la naturaleza de la profesión periodística, e intentar que, en la medida de lo posible, alcancen ese objetivo por sí mismos.

- Crear una atmósfera en la que incluso estas obligaciones básicas estén abiertas al debate.

- Ayudar a los alumnos para que sean capaces de reconocer y articular sus propios sistemas individuales de creencias morales y para que sean capaces de justificarlas.

- Facilitar las oportunidades para la exposición ante la clase de forma que las diversas teorías puedan ser discutidas por los propios compañeros y, en su caso, con periodistas en ejercicio, y también a través de lecturas relativas a las teorías sobre la función de la prensa, el análisis de casos prácticos y la filosofía moral.

- Cuestionar las creencias de los alumnos a través de las discusiones en clase, tanto libres como dirigidas.

- Evaluar la interpretación del alumno a través de la comparación con la toma de decisiones que han tomado periodistas expertos en situaciones similares.

- Crear modelos de evaluación tan claros como sea posible, e intentar involucrar a los propios alumnos en la elaboración de los mismos. 
Se podría alegar que la relación de responsabilidades es demasiado exhaustiva, así como que algunas de las recomendaciones expuestas resultan en cierto modo utópicas. En todo caso, parece razonable pensar que la mayoría de ellas servirán para evitar en la medida de lo posible del adoctrinamiento y enseñar la asignatura de forma que el futuro profesional salga mejor preparado desde todos los puntos de vista y, en especial, más concienciado respecto a las cuestiones relacionadas con la Ética.

\section{Metodología}

Los resultados de una investigación llevada a cabo en 14 países miembros de la Unión Europea (Alemania, Austria, Bélgica, Dinamarca, España, Finlandia, Francia, Gran Bretaña, Grecia, Irlanda, Italia, Países Bajos, Portugal y Suecia) con el objetivo de conocer la realidad de la enseñanza de la asignatura en los centros de educación superior de todos ellos nos servirá para conocer en qué medida los profesores europeos de Ética Periodística son conscientes del riesgo del adoctrinamiento y qué metodología docente utilizan para tratar de evitarlo. En concreto, se recogieron las opiniones de 50 profesores encargados de impartir la asignatura sobre diversas cuestiones, entre ellas las técnicas pedagógicas que suelen emplear en sus clases. Para ello, se elaboró un listado a partir de los distintos estudios previos de carácter similar que se han realizado en Estados Unidos (Braun, 1999; Christians y Covert, 1980; Lambeth, Christians y Cole, 1994; Van Horn, 1997), aunque con elementos modificados, añadidos o suprimidos, hasta incluir las siguientes:

- Análisis de casos prácticos

- Discusiones en pequeños grupos

- Estudio en profundidad de temas seleccionados

- Explicación de principios deontológicos

- Invitación a docentes de otros departamentos

- Invitación a profesionales ajenos a la institución

- Lecciones magistrales

- Lecturas obligatorias de libros y/o artículos

- Mesas redondas y seminarios

- Paneles de discusión

- Presentación oral de trabajos individuales

- Presentación oral de trabajos en grupo

- Proyección de diapositivas

- Proyección de películas

- Proyección de programas de televisión

- Proyección de transparencias

- Realización de entrevistas a profesionales de prestigio

- Realización de ensayos y ejercicios por escrito

- Role playing (juego de papeles) 
El estudio partió del supuesto de que la utilización de unos métodos no excluye el empleo de otros, sino que, justamente al contrario, se trata de procedimientos que tienen un carácter complementario, pues su empleo conjunto es adecuado y deseable para poder alcanzar una mayor variedad y por supuesto para captar el interés de los alumnos. Por ello, los docentes encuestados pudieron seleccionar un número indeterminado de técnicas sin tener que limitarse a un número máximo de ellas.

Por otro lado, el planteamiento de la investigación tuvo en cuenta el hecho de que las horas de clase y la duración del curso son limitadas. En consecuencia, resulta prácticamente imposible poder emplear todas las técnicas incluidas en el listado con cierta frecuencia, de manera que se consideró oportuno establecer una escala de categorías compuesta por cuatro grados que hacen referencia a la mayor o menor utilización de cada una de ellas. Por tanto, los profesores encuestados detallaron si emplean los métodos "muy frecuentemente"; "frecuentemente"; "con poca frecuencia"; y "nunca".

En este caso nos centraremos en analizar el empleo que realiza el profesorado de las técnicas antes mencionadas: el análisis de casos prácticos, las discusiones en pequeños grupos, y el role playing (juego de papeles). Con ello se pretende dar respuesta a los dos grandes objetivos de esta investigación: saber si los profesores de Ética de la Comunicación de los países mencionados tienen en cuenta a la hora de plantear la asignatura el peligro que supone el adoctrinamiento y conocer si la metodología docente que utilizan en sus clases es la más adecuada para poder evitar ese riesgo.

\section{Resultados ${ }^{1}$}

Los profesores europeos de Ética de la Comunicación apuestan preferentemente por dos técnicas pedagógicas y emplean de manera muy desigual los métodos más apropiados para salvar el riesgo de incurrir en el adoctrinamiento. En concreto, el estudio de casos prácticos y las lecturas obligatorias de libros y/o artículos son los dos métodos más empleados por los encuestados, pues el 90 y el $86 \%$ de ellos respectivamente (Tabla 1: Técnicas pedagógicas utilizadas) las utilizan de manera frecuente o muy frecuente. Las dos siguientes técnicas en el orden de preferencia, la explicación y desarrollo de principios deontológicos y la realización de ensayos y ejercicios por escrito obtienen un respaldo menor, un $72 \%$ cada una, sumando los encuestados que las emplean "muy frecuentemente" y "frecuentemente".

Tabla 1: Técnicas pedagógicas utilizadas (en \%)

\begin{tabular}{|l|c|c|c|c|}
\hline & Muy frec. & Frec. & Poco frec. & Nunca \\
\hline Libros y artículos & 58 & 28 & 8 & 6 \\
\hline Estudio casos & 54 & 36 & 6 & 4 \\
\hline Principios deontológicos & 36 & 36 & 12 & 16 \\
\hline
\end{tabular}




\begin{tabular}{|l|c|c|c|c|}
\hline Ensayos y ejercicios escritos & 32 & 40 & 12 & 16 \\
\hline Discusiones & 30 & 28 & 26 & 16 \\
\hline Presentaciones grupo & 28 & 14 & 24 & 34 \\
\hline Transparencias & 26 & 24 & 22 & 28 \\
\hline Trabajos individuales & 26 & 20 & 20 & 34 \\
\hline Temas seleccionados & 22 & 36 & 26 & 16 \\
\hline Lecciones magistrales & 16 & 20 & 22 & 42 \\
\hline Profesionales ajenos & 12 & 26 & 36 & 26 \\
\hline Diapositivas & 12 & 12 & 24 & 52 \\
\hline Programas TV & 10 & 42 & 22 & 26 \\
\hline Entrevistas a profesionales & 8 & 14 & 28 & 50 \\
\hline Seminarios & 6 & 38 & 18 & 38 \\
\hline Películas & 6 & 32 & 28 & 34 \\
\hline Paneles discusión & 6 & 32 & 20 & 42 \\
\hline Role playing & 4 & 10 & 34 & 52 \\
\hline Docentes otros departamentos & 4 & 8 & 42 & 46 \\
\hline
\end{tabular}

Fuente: Elaboración propia

Estos resultados son coincidentes en parte con los obtenidos en los diferentes estudios de este tipo llevados a cabo en Estados Unidos. Así, la investigación de Lambeth, Christians y Cole (1994) determinó que las dos técnicas más frecuentemente utilizadas eran el estudio de casos y las lecciones magistrales, que obtuvieron un $98,2 \%$ y un $93,3 \%$ de respaldo respectivo. Eso sí, conviene tener presente que ese estudio no estableció una escala con distintos grados de empleo de las técnicas pedagógicas. Por el contrario, la lectura de libros y artículos no aparecía entre los métodos más valorados.

Los datos del estudio de 1994 mostraban del mismo modo una coincidencia con los del primer estudio de hace más de 30 años (Christians y Covert, 1980), aunque reflejaban un cierto grado de evolución a favor del estudio de casos, que en aquella primera investigación sólo obtenía el 70\%, mientras que las lecciones magistrales eran la técnica mejor considerada, aunque con un porcentaje, $87 \%$, menor que en el sondeo de 1994.

Por lo que respecta a otras técnicas especialmente apropiadas para evitar el adoctrinamiento, los profesores europeos de la asignatura también emplean con cierta frecuencia (58\% de uso frecuente o muy frecuente) la discusión en pequeños grupos, que se sitúa como el quinto método en cuanto a popularidad entre el personal docente.

Asimismo, las técnicas que obtienen un peor resultado son el role playing y la invitación a docentes de otros departamentos, ya que el $86 \%$ de los encuestados afirma que emplea una y otra técnica con poca o ninguna frecuencia. Tampoco disfrutan de demasiada popularidad el empleo de diapositivas y la realización de entrevistas a profesionales de prestigio, que alcanzan el $78 \%$ en los porcentajes relativos a poco o ningún uso. 
Especialmente sorprendente parece el resultado obtenido por el role playing, técnica muy popular tradicionalmente en Estados Unidos (Silvia, 1992) y que fue valorada en el segundo de los estudios estadounidense con un 59,8\% (Lambeth, Christians y Cole, 1994). El resultado de esta técnica en el primer estudio era mucho más negativo, con un 12\% (Christians y Covert, 1980). Por ello, hasta cierto punto era lógico pensar que se trataba de una técnica pedagógica cada vez más empleado. Sin embargo, los datos obtenidos en la presente investigación ponen en entredicho esa conclusión, al menos en el ámbito geográfico de la Unión Europea.

No obstante, cabe matizar que los profesores encuestados tuvieron la oportunidad de apuntar posibles acciones que, en su opinión, sean susceptibles de ser puestas en marcha con el objetivo de mejorar la enseñanza de la asignatura. Una de las sugerencias más comunes fue la de poder disponer de un mayor número de horas, un factor que repercutiría muy positivamente en el uso de algunas técnicas pedagógicas que no tenían cabida en el curso, entre las cuales mencionaron precisamente el role playing.

Del mismo modo, algunos de los docentes abogaron por la idoneidad de contar con grupos más pequeños, lo que igualmente facilitaría el desarrollo de algunos de los métodos pedagógicos, empezando precisamente por el estudio de casos prácticos.

\section{Conclusiones y prospectiva}

Los profesores europeos de Ética y Deontología de la Comunicación parecen tener presente el riesgo del adoctrinamiento a la hora de escoger las técnicas pedagógicas que emplean en sus clases, a juzgar por el protagonismo que otorgan al análisis de casos prácticos $\mathrm{y}$, en general, por aquellas que promueven la participación activa del alumnado. El empleo de otras técnicas complementarias, como el role playing o los grupos de discusión está muy condicionado por la limitación temporal que supone la duración del curso.

De cara al futuro, sería interesante llevar a cabo un estudio similar pero referido a los alumnos en lugar de a los profesores, para tener en cuenta su punto de vista y comprobar cómo influye la asignatura en la manera en la que analizan un caso real y, en un hipotético futuro, en el modo en el que toman una decisión. En ese sentido, un reciente estudio llevado a cabo de Estados Unidos (Walker, 2011) resalta la tremenda importancia de la asignatura en este sentido, como herramienta fundamental para contar con muchos más elementos de juicio y con un criterio mucho más claro a la hora de decidir. Quedaría, por tanto, comprobar si en el ámbito geográfico concreto de la Unión Europea sucede lo mismo y sería igualmente interesante constatar si hay algún tipo de diferencia en función del país concreto, del sistema educativo o del contexto o circunstancias propias de cada uno de ellos. 


\section{Referencias bibliográficas}

ARISTÓTELES (1988). Ética nicomáquea. Madrid: Gredos.

BLACK, J. (1992). Media Ethics. En MURRAY, M. D. y FERRI, A. J. (eds.). Teaching Mass Communication. A Guide to Better Instruction. New York: Praeger, p. 235-255.

BRAUN, M. J. (1999). "Media Ethics Education: A Comparison of Student Responses". En Journal of Mass Media Ethics, vol. 14, n 3, pp. 171-182.

CHRISTIANS, C. G. y COVERT, C. L. (1980). Teaching Ethics in Journalism Education. New York, Hastings-on-Hudson: The Hasting Center.

COBO, J. M. (2001). Ética profesional en ciencias humanas y sociales. Madrid: Huerga Fierro Editores.

DÍAZ DEL CAMPO, J. (2012). "Técnicas pedagógicas básicas para la enseñanza de la ética periodística". En Etic@net, no 121. http://www. grupoteis.com/revistaindex.php /eticanet/article/view/13. [8 de julio de 2013].

DÍAZ DEL CAMPO, J. (2012). "Objetivos pedagógicos básicos en la enseñanza de la ética de la comunicación". En Vivat Academia, no 12, vol. 2. http://www.vivatacademia. net/ numeros/n121/DATOSS 121.htm\#objetivos. [ 7 de julio de 2013].

ELLIOT, D. (1984). Toward the Development of a Model for Journalism Ethics Instruction. Ph. D. dissertation, Harvard University.

ETXEBERRÍA, X. (2000). Teaching Professional Ethics. En DE STEXHE, G. y VERSTRAETEN, J. (eds.). Matter of Breath: Foundations for Professional Ethics. Lovaina: Peeters.

THE HASTINGS CENTER (1980). The Teaching of Ethics in Higher Education. New York: Plenum Press.

LAMBETH, E., CHRISTIANS, C. y COLE, K. (1994). "Role of the Media Ethics Course in the Education of Journalists". En Journalism Educator, vol. 49, n 3 , pp. 20-26.

LINDE NAVAS, A. (2009). "Teorías y procedimientos de educación moral en ética y deontología de la comunicación”. En Comunicación y Sociedad, vol. 22, n 2 , pp. 35-58.

MERRILL, J. C. (1978). "Do we teach ethics - Or do we teach "about" ethics". En Journalism Educator, vol. 33, n ${ }^{\text {2 }}$, p 59.

MERRILL, J. (2002). Journalism ethics in twilight. Conferencia pronunciada en el seminario Global Media Ethics. Universidad de Tampere, Finlandia, 26 de marzo.

SILVIA, T. (1992). "Teaching ethics: A model for role playing". En Feedback, vol. $33, n^{\circ} 4$, pp. 22-23.

VAN HORN, T. L. (1997). Teaching Ethics in Communication Courses. Ph. D. dissertation. Fullerton, Faculty of California State University.

WALKER, M. (2011). 'Evaluating the intervention of an ethics class in students' ethical decision-making". En Journal of the Scholarship of Teaching and Learning, vol. $11, n^{\circ} 4$, pp. $69-89$. 


\section{Notas}

1 Parte de los argumentos que se exponen en este epígrafe pueden encontrarse también en DÍAZ DEL CAMPO, Jesús (2012): Técnicas pedagógicas básicas para la enseñanza de la ética periodística, en Etic@net, no 121. Disponible en: http://www. grupoteis.com/ revista/index.php/eticanet/article/ view/13. [8 de julio de 2013]

\section{El autor}

Jesús Díaz del Campo es licenciado en Periodismo y doctor en Comunicación por la Universidad Complutense de Madrid. Actualmente es profesor en la Universidad Internacional de La Rioja, donde imparte las asignaturas Ética y Deontología de la Comunicación; Comunicación Radiofónica; Locución Radiofónica y Presentación Televisiva; y Comunicación Oral y Escrita. Profesionalmente ha ejercido como periodista en diversos medios e instituciones durante más de 15 años. 